\title{
Polymerase mutations rtN238R, rtT240Y and rtN248H of hepatitis $B$ virus decrease susceptibility to adefovir
}

\author{
QIN Bo ${ }^{1,2,3}$, PEI RongJuan ${ }^{1}$, HE TingTing ${ }^{2}$, HUANG ZhaoHui ${ }^{2}$, PAN GuoShao $^{2}$, \\ TU ChunYu ${ }^{2}$, LU MengJi ${ }^{1,3}$ \& CHEN XinWen ${ }^{1 *}$ \\ ${ }^{1}$ State Key Laboratory of Virology, Wuhan Institute of Virology, Chinese Academy of Sciences, Wuhan 430071, China; \\ ${ }^{2}$ Shaoxing Centre for Disease Control and Prevention, Shaoxing 321071, China; \\ ${ }^{3}$ Institute of Virology, University Hospital of Essen, Essen 45122, Germany
}

Received September 17, 2012; accepted December 10, 2012; published online April 9, 2013

\begin{abstract}
Long term antiviral therapy with nucleos(t)ide analogs (NAs) may lead to the emergence of drug-resistance viral mutants in chronic hepatitis B virus (HBV) patient. The purpose of this study was to identify adefovir dipivoxil (ADV) resistance mutations of HBV polymerase and determine effective drugs to replace ADV. The reverse transcriptase (RT) coding region was PCR-amplified using HBV DNA extracted from patient blood samples and sequenced. Nineteen substitution mutations were detected. Among them, rtN238R, rtT240Y and $\mathrm{rtN} 248 \mathrm{H}$ were often observed in patients receiving ADV administration. These three potential drug resistant sites were introduced into HBV replication-competent plasmids. The in vitro susceptibility of both wild-type (WT) and mutant-type (MT) HBV to NAs was analyzed by Southern blotting and quantitative real-time PCR. The rtN238R, rtT240Y and $\mathrm{rtN248H}$ substitutions had no obvious effect on HBV DNA replication or gene expression. The in vitro susceptibility analysis showed that $\mathrm{rtN238R}$, rtT240Y and $\mathrm{rtN} 248 \mathrm{H}$ substitutions were responsible for the reduced susceptibility to ADV, and demonstrated a 5.42-, 2.89- and 5.72-fold increase in resistance towards ADV, respectively. However, HBV harbored these mutations retained normal susceptibility to LMV, LdT, ETV and TDF.
\end{abstract}

hepatitis B virus, resistance mutation, adefovir dipivoxil (ADV)

Citation: Qin B, Pei R J, He T T, et al. Polymerase mutations rtN238R, rtT240Y and rtN248H of hepatitis B virus decrease susceptibility to adefovir. Chin Sci Bull, 2013, 58: 1760-1766, doi: 10.1007/s11434-013-5770-x

Hepatitis B virus (HBV) is a member of the hepadnavirus family and contains a $3.2 \mathrm{~kb}$, relaxed circular (RC), partially double-stranded DNA genome [1,2] with four overlapping open reading frames (ORFs): $\mathrm{P}, \mathrm{S}, \mathrm{C}$, and $\mathrm{X}$ [3]. HBV infection can lead to acute and chronic necroinflammatory liver diseases, especially hepatocellular carcinoma (HCC), which account for half a million to 1.2 million deaths each year in the more than 350 million people who are chronic carriers of HBV [4-7]. HBV replicates through reverse transcription of an RNA intermediate, called pregenomic RNA (pgRNA) [8]. Like retroviruses with an error-prone polymerase, such as human immunodeficiency virus (HIV), HBV has a quasispecies distribution in infected individuals

*Corresponding author (email: chenxw@wh.iov.cn)
[4,9]; virions with relevant resistance mutations can be selected after treatment with nucleos(t)ide analogue (NA) drugs.

To date, complete HBV eradication is seldom accomplished with currently available agents such as interferon and NAs [10]. Lamivudine (LMV), adefovir dipivoxil (ADV), entecavir (ETV), telbivudine (LdT) and tenofovir (TDF) are widely used for treatment of chronic hepatitis B (CHB) [11]. NA treatment inhibits HBV replication in patients and significantly reduces mortality, the risk of cirrhosis-related complications, and the incidence of hepatocellular carcinoma (HCC) [12]. However, the frequent emergence of drug-resistance mutations often leads to treatment failure and liver disease progression [13].

Various drug-resistant mutations of $\mathrm{HBV}$ have been identified, all of which were located in the reverse tran- 
scriptase (RT) region of HBV polymerase, which is further divided into seven domains: A-G [14]. Resistance to LMV and LdT is conferred by mutations in the YMDD motif within the $\mathrm{C}$ domain. The most common mutations are rtM204I/V [15], which are usually accompanied with compensatory mutations of $\mathrm{rtL} 180 \mathrm{M}$ and/or $\mathrm{rtV} 173 \mathrm{~L}$ to restore HBV replication capacity [12,16,17]. An $\mathrm{rtA} 181 \mathrm{~V} / \mathrm{T}$ or rtN236T substitution can reduce the anti-HBV effect of ADV [18]. Resistance to ETV requires a combination of mutations in the B, C, or D domain, such as rtI169T, rtM250I/V, and background substitutions at position rt204 $[12,19]$. However, there has been no reported TDF resistance mutation, except rtA194T which needs further confirmation [12,20-22].

The present study focused on the analysis of HBV polymerase sequence variation from $\mathrm{CHB}$ patients to detect novel potential drug-resistance mutations. Analysis of HBV sequences from $5 \mathrm{CHB}$ patients undergoing LMV and ADV alternate therapies identified many substitution mutations, including rtN238R, rtT240Y and rtN248H. These newly discovered mutations were then introduced into HBV replication-competent plasmids. In vitro analysis demonstrated that rtN238R, rtT240Y and rtN248H resulted in 5.61-, 2.76and 5.71-fold increases in resistance toward ADV, respectively. The results of the present study should provide certain specific references for clinical drug administration.

\section{Mateirals and methods}

(i) $\mathrm{CHB}$ patients and sequence analysis. Serum samples from $\mathrm{CHB}$ patients receiving NA therapy were collected, from which HBV DNA was isolated using the QIAamp DNA Blood Mini Kit (Qiagen, Hilden, Germany). The HBV-RT segment was PCR-amplified with the primer pair: RT-F and RT-R (Table 1), and cloned into the pCR2.1 vector. Five clones of each PCR product were two-way sequenced

Table 1 Primer sequences for the construction of MT HBV plasmids and qRT-PCR ${ }^{\text {a) }}$

\begin{tabular}{llc}
\hline \multicolumn{1}{c}{ Name } & \multicolumn{1}{c}{ Sequence $\left(5^{\prime} \rightarrow 3^{\prime}\right)$} & $\begin{array}{c}\text { Amplicon } \\
\text { size }(\mathrm{bp})\end{array}$ \\
\hline RT-F & CTAGGACCCCTGCTCGTGTT & 843 \\
RT-R & CGCAAACCCCAAAAGACCCA & \\
Fusion-F & TCTTCTCGAGGATTGGGGACC & 1257 \\
Fusion-R & GCAGCCATGGAAACGATGTAT & 552 \\
rtN238R-F & CATTTA AACCCTCGCAAAACA AAG & 730 \\
rtN238R-R & CTTTGTTTTGCGAGGGTTTAAATG & 548 \\
rtT240Y-F & TAAACCCTAACAAATACAAGAGATG & 735 \\
rtT240Y-R & CATCTCTTGTATTTGTTAGGGTTTA & 523 \\
rtN248H-F & GGGTTACTCTCTACATTTTATGGGT & 760 \\
rtN248H-R & CATAAAATGTAGAGAGTAACCCCTA & 100 \\
HBV-RC-F & GTTGCCCGTTTGTCCTCTAATTC & \\
HBV-RC-R & GGAGGGATACATAGAGGTTCCTT & \\
\hline
\end{tabular}

a) MT, mutant; qRT-PCR, quantitative real-time PCR; F, forward; R, reverse; bold text, italics: substitution mutation for mutant $\mathrm{HBV}$ plasmids construction and aligned with WT HBV (GenBank accession no. X02763.1, genome type A, subtype $a d w 2$ ).

(ii) Plasmid constructs. HBV mutants (MTs) were constructed using fusion-PCR with the primers listed in Table 1 carrying aimed mutations and pHBV1.3 as a template, which is a replication-competent plasmid including 1.3-fold over-length HBV genotype A2 genome (GenBank accession no. X02763.1, subtype $a d w 2$ ) [23,24], with a pBluescript KS+backbone.

(iii) NAs. NAs including 2',3'-dideoxy-3'-thiacytidine (LMV; Glaxo Smith Kline, Brentford, Middlesex, UK), ADV (Gilead Sciences, Foster City, CA, USA), ETV (BristolMyers Squibb Co., New York, NY, USA), LdT (Novartis Pharmaceuticals Canada Inc., Dorval, Quebec, Canada) and TDF (Gilead Sciences) were diluted according to the manufactures' instructions and used in the assays at the indicated concentrations.

(iv) Cell culture and transfection. Hepatocellular carcinoma Huh7 cells were cultured in Dulbecco's modified Eagle's medium (DMEM; Invitrogen, Carlsbad, CA, USA) at $37^{\circ} \mathrm{C}$ in a $5 \% \mathrm{CO}_{2}$ atmosphere supplemented with $10 \%$ fetal bovine serum (FBS; Gibco, Carlsbad, CA, USA), 2 mmol L ${ }^{-1}$ of glutamine, $100 \mathrm{IU} \mathrm{mL}^{-1}$ of penicillin and 100 IU m L $\mathrm{m}^{-1}$ of streptomycin [25]. Huh7 cells were seeded in 6 -well plates $\left(1 \times 10^{6} /\right.$ well $)$ and transfected with $2 \mu \mathrm{g} \mathrm{HBV}$ bearing plasmid per well using lipofectamine 2000 (Invitrogen) with or without the indicated NA concentrations. For transfection experiments, HBV-bearing plasmids were co-transfected with a SEAP expression vector into Huh7 cells, the relative efficiency of transfections was assessed by measuring SEAP activity in the culture medium using a chemiluminescent detection method [26].

(v) Enzyme-linked immunosorbent assay (ELISA). Huh7 cells were transfected with the indicated HBV-bearing plasmids, hepatitis B surface antigen (HBsAg) and hepatitis B e antigen $(\mathrm{HBeAg})$ in the supernatant at $96 \mathrm{~h}$ post transfection (hpt) were detected using a diagnostic kit (Shanghai Kehua Diagnostic Medical Products Co., Ltd., Shanghai, China) according to the manufacturer's instructions [25].

(vi) Western blotting. Huh7 cells were transfected with the indicated HBV-bearing plasmids and harvested at $96 \mathrm{hpt}$. The protein concentrations were determined using the BioRad protein assay kit (Bio-Rad Laboratories, Hercules, CA, USA). Total cell lysates $(50 \mu \mathrm{g})$ from the transfected cells were assayed by Western blotting by probing with an anti-hepatitis B core antigen ( $\mathrm{HBcAg}$ ) antibody (Dako Cytomation Co., Carpinteria, CA, USA) and anti- $\beta$-actin (Beyotime Biotech Inc., Nantong, China), and detected by chemiluminescence (SuperSignal West Pico; Thermo Scientific-Pierce Protein Biology Products, Rockford, IL, USA) followed by autoradiography and densitometry analysis using a secondary goat anti-mouse IgG HRP antibody (Jackson ImmunoResearch Laboratories, Inc., West Grove, PA, USA; diluted 1:10000) $[27,28]$. 
(vii) Analysis of HBV DNA from intracellular core particles by Southern blotting. Replication competent HBV WT/MTs plasmids were transfected into Huh7 cells. Total HBV DNA replicative intermediates from intracellular core particles were extracted and subjected to agarose gel electrophoresis, followed by denaturation and Southern blotting with a ${ }^{32} \mathrm{P}$-labeled full length HBV probe according to previously published protocols [25,29]. Hybridization signals were visualized and analyzed using a phosphoimager (Cyclone, Packard Instrument Co., Inc., Meriden, CT, USA). Data were quantified with OptiQuant software (Packard Bioscience Co., Inc., Meriden, CT, USA).

(viii) Quantitative real-time PCR (qRT-PCR). Cell lysates of Huh7 were treated with DNase I (Roche) at $37^{\circ} \mathrm{C}$, 30 min to digest input plasmid DNA. Total HBV DNA replicative intermediates were purified from lysates of Huh7 cells $96 \mathrm{hpt}$ and were used as templates for qRT-PCR, which was conducted using SYBR Green I nucleic acid stain (Roche Diagnostics GmbH, Mannheim, Germany) on a Light Cycler real-time thermal cycler (Applied Biosystems, Foster City, CA, USA) according to the manufacturers' instructions [29]. Primers HBV-RC-F and HBV-RC-R (Table 1) hybridized to the HBV surface gene were designed to quantify HBV-DNA RC genomes (100 bp fragment) by qRT-PCR relative to an external plasmid DNA standard [30].

(ix) Statistical analysis. The statistical analysis was carried out using GraphPad (GraphPad Software, San Diego, USA). Differences in multiple comparisons were determined for statistical significance using Student's $t$-test. $P<$ 0.05 was considered as statistically significant. Results are presented as means \pm SD.

\section{Results}

\subsection{Sequence analysis of HBV-RT from CHB patients}

Five patients with lengthy $\mathrm{CHB}$ histories ( $>3$ years) took part in this study. An increase in serum alanine aminotransferase (ALT) and HBV DNA levels indicated HBV variation. When resistance mutations to LMV or ADV (rtM204V/I for $\mathrm{LMV}, \mathrm{rtA} 181 \mathrm{~T} / \mathrm{V}$ and $\mathrm{rtN} 236 \mathrm{~T}$ for $\mathrm{ADV}$ ) appeared along with the virologic breakthrough, which normally happened in 6-12 months treatment, a conversion therapy was carried out. The HBV-RT segment was PCR-amplified using HBV DNA extracted from the serum sample as a template and sequenced. Sequence alignments with a reference indicated a variety of quasispecies in $\mathrm{CHB}$ patients after NA treatment, including rtS21A, rtL122F, rtN124F/H, rtP130Q, rtD131N, rtY135S/F, rtV142E, rtL145M, rtL180M rtA181V/T, rtS202G, rtM204V, rtF221Y, rtN236T, rtN238 R/H, rtT240Y, $\mathrm{rtN} 248 \mathrm{H}, \mathrm{rtE} 263 \mathrm{D}$ and $\mathrm{rtQ} 267 \mathrm{H}$ in the HBV-RT sequence (Figure 1) [31-33]. After LMV treatment, rtM204V and/or rtL180M mutations emerged as described in previous reports [12,16,17]. Interestingly, a novel mutation, rtQ267H/R, was occasionally found after LMV therapy. Other than rtA181V and rtN236T, which occurred in all samples after about 12 months of ADV administration, we also commonly found the mutations $\mathrm{rtN} 238 \mathrm{R} / \mathrm{H}$, rtT240Y and $\mathrm{rtN} 248 \mathrm{H}$, even though they did not appear in all samples (Figure 1). The frequencies of $\mathrm{rtN} 238$ and $\mathrm{rtN} 248(80 \%)$ substitutions were higher than that of $\mathrm{rtT} 240(40 \%)$ in different patients (Figure 1). The relatively high prevalence of these three substitutions in $\mathrm{CHB}$ patients with ADV therapy implies that they might be selected after ADV long-term usage and attenuate the anti-HBV effect of ADV.

\subsection{The replication capacity of MT pHBV1.3 in vitro}

To further analyze the influence of the rtN238, rtT240 and rtN248 mutations on HBV replication and sensitivity to ADV, three MT HBV replication-competent plasmids, pHBV-rtN238R, -rtT240Y and -rtN248H, were constructed based on pHBV1.3 [34]. The replication-competent plasmids were then transfected into Huh7 cells and pHBV1.3 was used as a control. Southern blotting (upper panel) and subsequent densitometry analysis (lower panel) revealed no significant difference between the MT and parent HBV (Figure 2). There was a slightly lower HBV DNA level in MTs compared with the WT as determined by qRT-PCR, but they were not statistically different (Figure 2(b)). Thus, none of the substitutions had any obvious effect on HBV replication.

HBsAg and HBeAg levels in culture supernatant at 96 hpt were also measured by ELISA (Figure 2(c)), while $\mathrm{HBcAg}$ in cell lysates was analyzed by Western blotting (Figure 2(d)). The results indicated that the recombinant HBV with the substitution of rtN238R, rtT240Y or rtN248H, respectively, had similar expression levels as $\mathrm{HBsAg}$, $\mathrm{HBeAg}$ and $\mathrm{HBcAg}$ with that of HBV WT. Taken together, the rtN238R, rtT240Y and rtN248H substitutions had no obvious effect on HBV DNA replication or gene expression under our experimental conditions.

\section{3 rtN238R, rtT240Y and rtN248H in HBV substitu- tions are involved in reduced susceptibility to ADV}

In order to determine whether rtN238R, rtT240Y and rtN248H influenced HBV susceptibility to LMV and ADV, Huh7 cells were transfected with MT or WT HBV plasmids, then exposed to the indicated NA concentrations and the core-associated DNA was analyzed by Southern blotting. As shown in Figure 3(a), LMV inhibited the replication of MT and WT HBV in a dose-dependent manner. In contrast, ADV reduced WT HBV replication in a dose-dependent manner, but did not influence the three MT HBVs, indicating that MTs were less susceptible to ADV (Figure 3(b)).

qRT-PCR targeted to HBV RC DNA was performed to calculate the increase in resistance of $\mathrm{rtN} 238 \mathrm{R}, \mathrm{rtT} 240 \mathrm{Y}$ and $\mathrm{rtN} 248 \mathrm{H}$ to $\mathrm{ADV}$. The half maximal effective concentration 

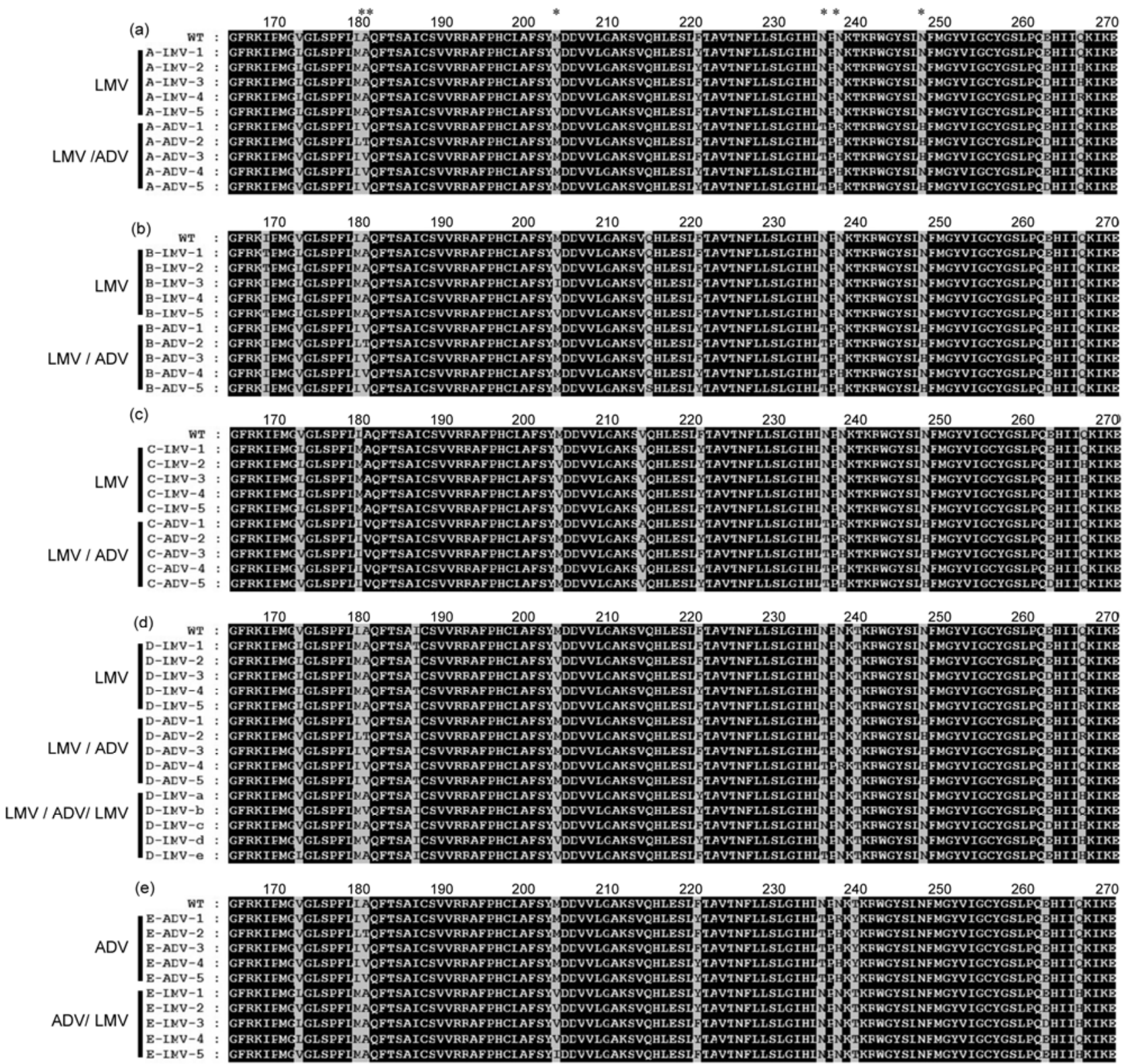

Figure 1 Sequence alignments of HBV RT regions. HBV DNA was isolated from serum samples of CHB patient (a), (b), (c), (d) and (e) who treated alternately with LMV and ADV when the drug-resistance showed up. The HBV-RT segments were PCR-amplified and cloned. Five clones were sequenced for each sample. Then the sequences were aligned with the reference sequence (WT, GenBank accession no. X02763.1, genotype A). The region (rt167 to rt270) contained the substitution sites are shown.

$\left(\mathrm{EC}_{50}\right)$ of pHBV1.3-WT, -rtN238R, -rtT240Y and -rtN248H to ADV were about $1.38,7.49,3.99$ and $7.90 \mu \mathrm{mol} \mathrm{L} \mathrm{L}^{-1}$, respectively, as calculated by qRT-PCR (Figure 4(b)), which was much higher than that of WT $\left(1.38 \mu \mathrm{mol} \mathrm{L} \mathrm{L}^{-1}\right)$. Their resistance indexes (the $\mathrm{EC}_{50}$ value of $\mathrm{MT}$ divided by that of WT) to ADV were about 5.42, 2.89 and 5.72, respectively.

2.4 rtN238R, rtT240Y and rtN248H substitutions maintained normal susceptibility to other NAs, but not ADV

rtN238R, rtT240Y and rtN248H substitutions reduced the
anti-HBV effect of ADV. We further analyzed their susceptibility to other NAs. Huh7 cells were transfected with pHBV-rtN238R, -rtT240Y, -rtN248H or pHBV1.3 and then treated with indicated concentrations of LMV, ETV, LdT and TDF. qRT-PCR was used to measure the HBV RCDNA copy number and the $\mathrm{EC}_{50}$ of WT and MT HBV to LMV (Figure 4(a)), ADV (Figure 4(b)), LdT (Figure 4(c)), ETV (Figure 4(d)) and TDF (Figure 4(e)). The EC $_{50}$ values of pHBV-rtN238R, -rtT240Y, -rtN248H to LMV, LdT, ETV and TDF were all close to that of WT $(1.08,11.5,0.79$ and $0.195 \mu \mathrm{mol} \mathrm{L} \mathrm{L}^{-1}$, respectively). The results indicated that rtN238R, rtT240Y and rtN248H decreased the susceptibility 

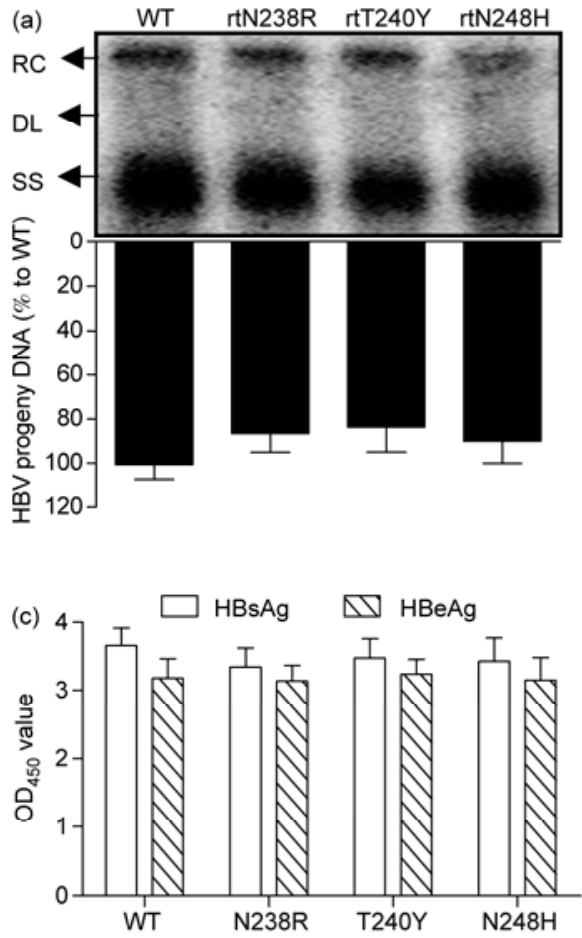

(b)

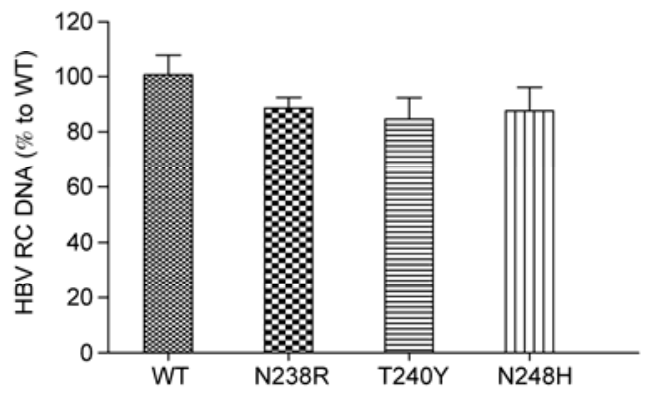

(d)

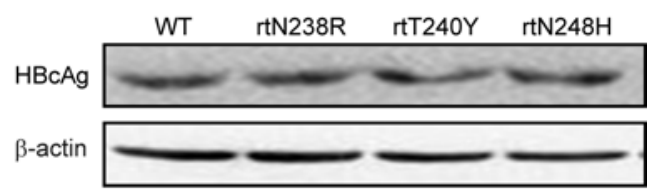

Figure 2 Replication capacity and antigen expression levels of WT and HBV mutants. (a) Huh7 cells were transfected with WT or MT HBV plasmids. Encapsulated viral DNA was extracted and detected by Southern blotting (upper panel). Relaxed circular (RC), double stranded linear (DL) and single stranded (SS) HBV DNAs are indicated. The relative level of WT HBV replication capacity is shown as a percentage of the control by gray analysis (lower panel). (b) HBV RC DNA of mutants compared with that of WT HBV is shown as a percentage of the control. Each value is the mean of at least 3 independent experiments. The error bars represent the standard deviation (SD). (c) HBsAg and HBeAg in the supernatant were detected using an ELISA diagnostic kit (Shanghai Kehua Diagnostic Medical Products Co., Ltd.) according to the manufacturer's instructions. (d) Total lysates of Huh7 cells were prepared and $50 \mu \mathrm{g}$ of total protein was subjected to Western blotting. $\mathrm{HBcAg}$ was detected using mouse polyclonal antibody (upper panel) and $\beta$-actin was used as a loading control (lower panel).
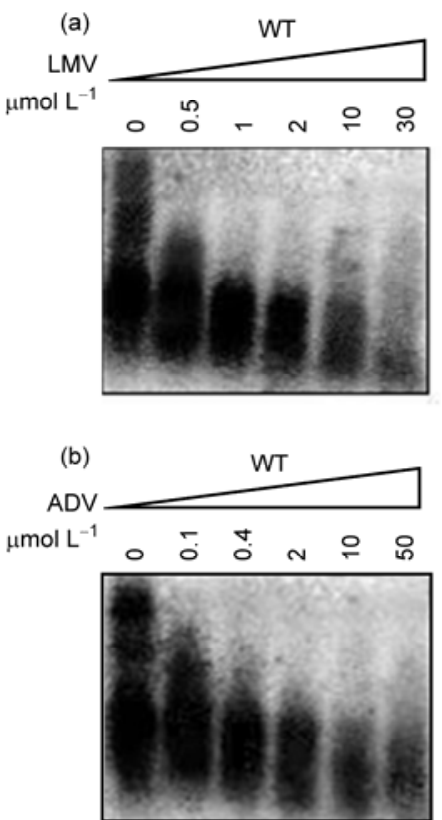
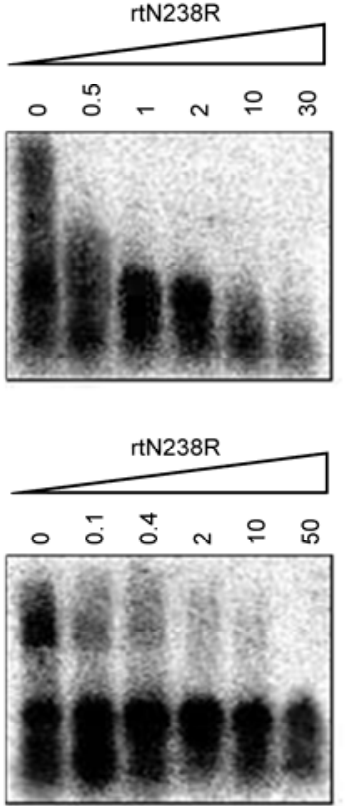
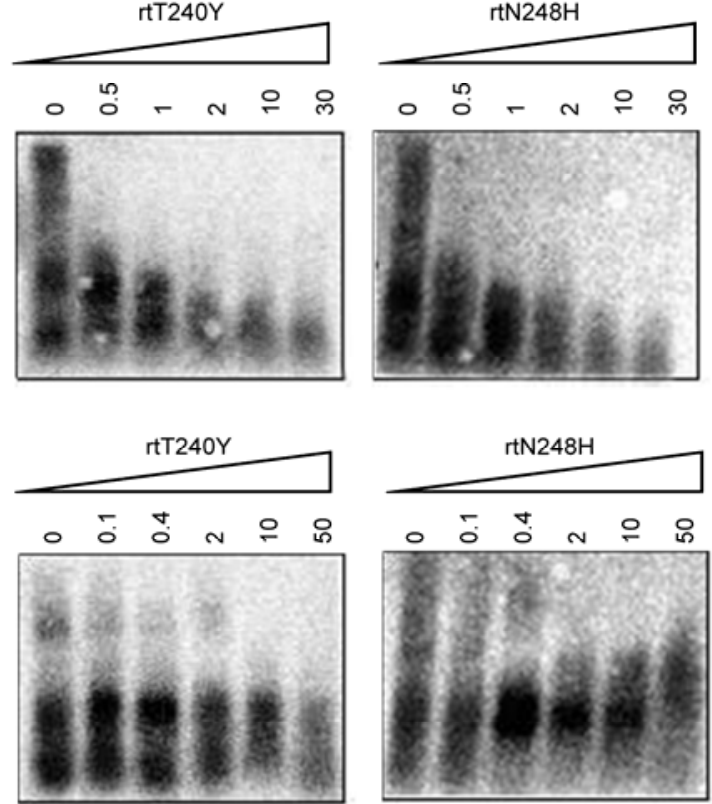

Figure 3 Southern blot analysis was used to detect the anti-HBV effect of LMV and ADV. Huh7 cells were transfected with pHBV1.3, pHBV-rtN238R, $-\mathrm{rtT} 240 \mathrm{Y}$ and $-\mathrm{rtN} 248 \mathrm{H}$, and treated with LMV (a) and ADV (b) at the indicated concentrations. Core-associated HBV DNA was extracted from the cell lysates $96 \mathrm{hpt}$ and subjected to agarose gel electrophoresis, followed by denaturation and Southern blotting. 
of HBV to ADV, but they were still susceptible to LMV, LdT, ETV and TDF in vitro.

\section{Discussion}

HBV infection is a worldwide health problem. Chronic HBV infection can be treated with NAs, which are also widely used for HIV and herpes viruses [35,36], but drug resistance severely impaired HBV therapy. In the present study, we identified resistance mutations of $\mathrm{HBV}$ to ADV, which has been widely used together with LMV. Among the mutations found in patient blood samples, rtL180M, rtA181V/T, rtS202G, rtM204V/I, and rtN236T were confirmed as NA resistance mutations, whereas $\mathrm{rtS} 21 \mathrm{~A}, \mathrm{rtL} 122 \mathrm{~F}$, rtN124F/H, rtP130Q, rtD131N, rtY135S/F, rtV142E, rtL145M, $\mathrm{rtF} 221 \mathrm{Y}$, rtE263D and $\mathrm{rtQ} 267 \mathrm{H}$ were found in CHB patients [31-33,37,38] . Moreover, rtN238R, rtT240Y and rtN248H were regularly turned up in CHB patients with ADV treatment, and they were introduced into replication- competent plasmids to demonstrate that they were also responsible for reduced susceptibility to ADV in vitro.

NAs that mimic physiological nucleosides (dA/T/G/CTP) in terms of uptake and metamorphosis are incorporated into newly synthesized viral DNA giving rise to synthesis inhibition and termination [39]. ADV showed comparative antiviral activity against LMV-resistant HBV mutants and WT HBV [40]. However, in the first year of treatment, ADV resistance emerged in about $20 \%$ of LMV-resistant patients and in about $2 \%$ of patients with no LMV resistance-associated mutations $[12,41]$, which may explain why so many
ADV resistance-associated mutations were selected and found in the present study. A higher resistance equates to weaker selective pressure; hence, the frequency of rtN238R and $\mathrm{rtN} 248 \mathrm{H}$ was higher than that of rtT240Y, in accordance with the clinical data. Overall, according to our data, $\mathrm{HBs} / \mathrm{e} / \mathrm{cAgs}$ were not directly associated with HBV replication in vitro.

HBV resistance mutations were all found in HBV-RT, with no exception for rtN238R (domain D), rtT240Y (domain D) and rtN248H (domain E). The HBV genome overlaps, as the $S$ gene is completely contained within the $P$ gene and mutations in the HBV polymerase coding region may confer NA resistance that usually affects the HBsAg. However, rtN238R, rtT240Y and rtN248H substitutions had no influence on $\mathrm{HBsAg}$ secretion, because they all reside after the stop codon of the $S$ gene.

According to the three-dimensional (3D) HBV-RT structure predicted by SWISS-MODEL (http://swissmodel. expasy.org/), an automated protein modeling server, rtN238R, rtT240Y and rtN248H are situated close to residue M204 in the YMDD motif (data not shown), which was found to be the catalytic center for DNA synthesis. Substitutions may change the RT structure, and then prevent ADV from entering the catalytic pocket or make ADV separate from the catalytic pocket easyly thereby reduce the anti-HBV effect of ADV.

Fortunately, HBV mutants with any one of the rtN238R, rtT240Y or rtN248H substitutions retained normal susceptibility to LMV, LdT, ETV and TDF. In further research, it will be interesting to test whether the combination of these three mutations enhances ADV resistance, and what will
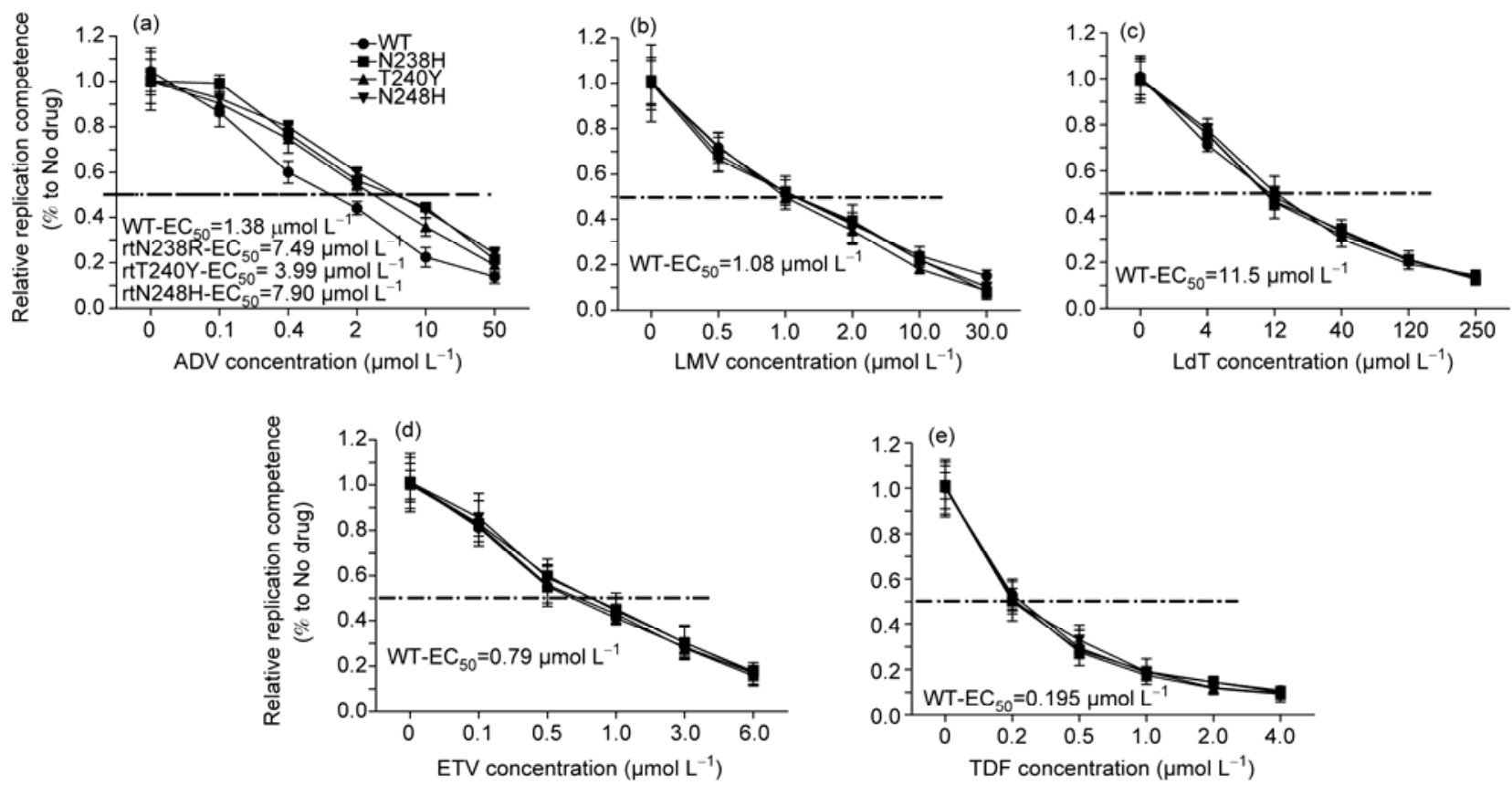

Figure 4 qRT-PCR was performed to detect the anti-HBV effect of 5 NAs. Huh7 cells were transfected with HBV-bearing plasmids, RC-DNA of encapsulated HBV DNA was extracted and analyzed by qRT-PCR to evaluate the antiviral effect of ADV (a), LMV (b), ETV (c), LdT (d) and TDF (e). The results shown were calculated as means \pm 2 SDs $(n=3)$. 
happen when they are combined with $\mathrm{rtA} 181 \mathrm{~V} / \mathrm{T}$ and/or rtN236T. Further, we plan to explore the susceptibility to NAs in hydrodynamically injected mice with replicationcompetent pAAV-HBV1.3.

We are grateful to the patients for providing the serum samples. This work was supported in part by the National Basic Research Program of China (2007CB512901).

1 Beck J, Nassal M. Hepatitis B virus replication. World J Gastroenterol, 2007, 13: 48-64

2 Gao W, Hu J. Formation of hepatitis B virus covalently closed circular DNA: Removal of genome-linked protein. J Virol, 2007, 81: 6164-6174

3 Mizokami M, Orito E, Ohba K, et al. Constrained evolution with respect to gene overlap of hepatitis B virus. J Mol Evol, 1997, 44 (Suppl 1): S83-90

4 Lee W M. Hepatitis B virus infection. N Engl J Med, 1997, 337: 1733-1745

5 Behal R, Jain R, Behal K K, et al. Seroprevalence and risk factors for hepatitis $\mathrm{B}$ virus infection among general population in northern India. Arq Gastroenterol, 2008, 45: 137-140

6 Mahoney F J. Update on diagnosis, management, and prevention of hepatitis B virus infection. Clin Microbiol Rev, 1999, 12: 351-366

7 Schildgen V, Ziegler S, Tillmann R L, et al. Novel mutation in YMDD motif and direct neighbourhood in a child with chronic HBVinfection and clinical lamivudine and adefovir resistance-A scholarly case. Virol J, 2010, 7: 167

8 Summers J, Mason W S. Replication of the genome of a hepatitis Blike virus by reverse transcription of an RNA intermediate. Cell, 1982, 29: 403-415

9 Gunther S, Fischer L, Pult I, et al. Naturally occurring variants of hepatitis B virus. Adv Virus Res, 1999, 52: 25-137

10 Yuen M F, Lai C L. Treatment of chronic hepatitis B. Lancet Infect Dis, 2001, 1: 232-241

11 Fung J, Lai C L, Seto W K, et al. Nucleoside/nucleotide analogues in the treatment of chronic hepatitis B. J Antimicrob Chemother, 2011, 66: $2715-2725$

12 Zoulim F, Locarnini S. Hepatitis B virus resistance to nucleos(t)ide analogues. Gastroenterology, 2009, 137: 1593-1608

13 Zoulim F. Antiviral therapy of chronic hepatitis B. Antiviral Res, 2006, 71: 206-215

14 Kwon H, Lok A S. Hepatitis B therapy. Nat Rev, 2011, 8: 275-284

15 Torresi J, Earnest-Silveira L, Civitico G, et al. Restoration of replication phenotype of lamivudine-resistant hepatitis B virus mutants by compensatory changes in the "Fingers" Subdomain of the viral polymerase selected as a consequence of mutations in the overlappings gene. Virology, 2002, 299: 88-99

16 Brunelle M N, Jacquard A C, Pichoud C, et al. Susceptibility to antivirals of a human HBV strain with mutations conferring resistance to both lamivudine and adefovir. Hepatology, 2005, 41: 1391-1398

17 Pallier C, Castera L, Soulier A, et al. Dynamics of hepatitis B virus resistance to lamivudine. J Virol, 2006, 80: 643-653

18 Osiowy C, Villeneuve J P, Heathcote E J, et al. Detection of rtN236T and $\mathrm{rtA} 181 \mathrm{~V} / \mathrm{T}$ mutations associated with resistance to adefovir dipivoxil in samples from patients with chronic hepatitis B virus infection by the INNO-LiPA HBV DR line probe assay (version 2). J Clin Microbiol, 2006, 44: 1994-1997

19 Leung N. Viral resistance in HBV infection: Diagnosis, implications and management. Trop Gastroenterol, 2008, 29: 123-128

20 Amini Bavil Olyaee S, Herbers U, Sheldon J, et al. The rtA194T polymerase mutation impacts viral replication and susceptibility to tenofovir in hepatitis B e antigen-positive and hepatitis B e antigennegative hepatitis B virus strains. Hepatology, 2009, 49: 1158-1165
21 Sheldon J, Camino N, Rodes B, et al. Selection of hepatitis B virus polymerase mutations in HIV-coinfected patients treated with tenofovir. Antivir Ther, 2005, 10: 727-734

22 Delaney W E, Ray A S, Yang H, et al. Intracellular metabolism and in vitro activity of tenofovir against hepatitis B virus. Antimicrob Agents Chemother, 2006, 50: 2471-2477

23 Wu C, Deng W, Deng L, et al. Amino acid substitutions at positions 122 and 145 of hepatitis B virus surface antigen (HBsAg) determine the antigenicity and immunogenicity of $\mathrm{HBsAg}$ and influence in vivo HBsAg clearance. J Virol, 2012, 86: 4658-4669

24 Lei Y C, Hao Y H, Zhang Z M, et al. Inhibition of hepatitis B virus replication by APOBEC3G in vitro and in vivo. World J Gastroenterol, 2006, 12: 4492-4497

25 Qiu J, Qin B, Rayner S, et al. Novel evidence suggests hepatitis B virus surface proteins participate in regulation of HBV genome replication. Virol Sin, 2011, 26: 131-138

26 Kain S R. Use of secreted alkaline phosphatase as a reporter of gene expression in mammalian cells. Methods Mol Biol, 1997, 63: 49-60

27 Pan X B, Wei L, Han J C, et al. Artificial recombinant cell-penetrating peptides interfere with envelopment of hepatitis $\mathrm{B}$ virus nucleocapsid and viral production. Antiviral Res, 2011, 89: 109-114

28 Guarnieri M, Kim K H, Bang G, et al. Point mutations upstream of hepatitis B virus core gene affect DNA replication at the step of core protein expression. J Virol, 2006, 80: 587-595

29 Meng Z, Xu Y, Wu J, et al. Inhibition of hepatitis B virus gene expression and replication by endoribonuclease-prepared siRNA. J Virol Methods, 2008, 150: 27-33

30 Untergasser A, Zedler U, Langenkamp A, et al. Dendritic cells take up viral antigens but do not support the early steps of hepatitis B virus infection. Hepatology, 2006, 43: 539-547

31 Santantonio T, Fasano M, Durantel S, et al. Adefovir dipivoxil resistance patterns in patients with lamivudine-resistant chronic hepatitis B. Antivir Ther, 2009, 14: 557-565

32 Margeridon Thermet S, Shulman N S, Ahmed A, et al. Ultra-deep pyrosequencing of hepatitis B virus quasispecies from nucleoside and nucleotide reverse-transcriptase inhibitor (NRTI)-treated patients and NRTI-naive patients. J Infect Dis, 2009, 199: 1275-1285

33 Milazzo L, Corbellino M, Foschi A, et al. Late onset of hepatitis B virus reactivation following hematopoietic stem cell transplantation: Successful treatment with combined entecavir plus tenofovir therapy. Transpl Infect Dis, 2012, 14: 95-98

34 Wu C, Zhang X, Tian Y, et al. Biological significance of amino acid substitutions in hepatitis B surface antigen (HBsAg) for glycosylation, secretion, antigenicity and immunogenicity of HBsAg and hepatitis B virus replication. J Gen Virol, 2010, 91: 483-492

35 Lok A S. Hepatitis B infection: Pathogenesis and management. J Hepatol, 2000, 32: 89-97

36 Jarvis B, Faulds D. Lamivudine. A review of its therapeutic potential in chronic hepatitis B. Drugs, 1999, 58: 101-141

37 Liu B M, Li T, Xu J, et al. Characterization of potential antiviral resistance mutations in hepatitis B virus reverse transcriptase sequences in treatment-naive chinese patients. Antiviral Res, 2010, 85: 512-519

38 Tan J, Degertekin B, Wong S N, et al. Tenofovir monotherapy is effective in hepatitis B patients with antiviral treatment failure to adefovir in the absence of adefovir-resistant mutations. J Hepatol, 2008, 48: 391-398

39 Galmarini C M, Mackey J R, Dumontet C. Nucleoside analogues: Mechanisms of drug resistance and reversal strategies. Leukemia, 2001, 15: 875-890

40 Cha C K, Kwon H C, Cheong J Y, et al. Association of lamivudineresistant mutational patterns with the antiviral effect of adefovir in patients with chronic hepatitis B. J Med Virol, 2009, 81: 417-424

41 Lee Y S, Suh D J, Lim Y S, et al. Increased risk of adefovir resistance in patients with lamivudine-resistant chronic hepatitis B after 48 weeks of adefovir dipivoxil monotherapy. Hepatology, 2006, 43: 1385-1391

Open Access This article is distributed under the terms of the Creative Commons Attribution License which permits any use, distribution, and reproduction in any medium, provided the original author(s) and source are credited. 